\title{
Bullous Pemphigoid and Percutaneous Endoscopic Gastrostomy
}

\author{
Tsukasa Nozu and Hiroaki Mita
}

\begin{abstract}
Background Bullous pemphigoid (BP) is an autoimmune subepidermal blistering disorder. It is rare in the general population but the prevalence was reported to be relatively high among the elderly in nursing homes. The aim of the study was to analyze the clinical characteristics and risk factor for BP in elderly patients with a long hospital stay.

Methods This was a retrospective survey with 36 hospitalized patients, aged 64 to 101 years, staying more than 2 years from July 2005 to April 2009. We encountered 5 newly occurring BP cases during this survey period and the remaining 31 patients were non-BP cases. All of these subjects were disabled elderly patients because of cerebral stroke, etc. We assessed the clinical variables as follows: age, sex, underling disease of hospitalization, length of hospital stay, feeding method, medication, tracheal tube indwelling and frequency of death and compared these 2 groups.

Results All of the patients underwent enteral feeding by percutaneous endoscopic gastrostomy (PEG) or nasogastric (NG) tube. In the univariate analysis, PEG and tube feeding (PEG and NG) were associated with BP $(\mathrm{p}<0.05)$. The multivariate logistic and Cox proportional hazard regression analysis were performed with age, sex (elderly female is well known feature of BP) and PEG or tube feeding, demonstrating only PEG was significantly and independently associated with BP $(\mathrm{p}<0.05)$.

Conclusion We found that PEG was significant risk factor for BP. Since the sample size was small in this survey, further study is required. Greater awareness of BP is needed for physicians managing institutionalized elderly patients with PEG.
\end{abstract}

Key words: enteral feeding, elderly patient, long hospital stay, prevalence, PEG

(Inter Med 49: 971-975, 2010)

(DOI: 10.2169/internalmedicine.49.3346)

\section{Introduction}

Bullous pemphigoid (BP) is a subepidermal blistering disorder mainly occurring in the elderly population, characterized by an autoimmune response to two hemidesmosomal proteins, designated BP 180 and BP $230(1,2)$. It is a rare disease in the general population and the one-year mortality is reported to be $19 \%$, which is more than twice that of the general population (3). On the other hand, the prevalence is relatively high among the elderly in nursing homes (4). Since management of these institutionalized elders is principally performed by physician, the diagnosis and treatment of $\mathrm{BP}$ is thought to be an important mission for not only der- matologists but also physicians. Moreover, a recent large cohort survey in United Kingdom revealed that the frequency of $\mathrm{BP}$ is increasing lately (3), suggesting their importance may become greater in the near future.

We encountered 5 BP cases among our hospitalized patients from July 2005 to April 2009. The aim of the study was to analyze clinical characteristics and risk factors for $\mathrm{BP}$ in patients with long hospital stay.

\section{Subjects and Methods}

This was a retrospective survey with 36 hospitalized patients, aged 64 to 101 years, staying more than 2 years from July 2005 to April 2009 (46 months). We encountered 5 
newly developed BP cases during this survey period and the remaining 31 patients without $\mathrm{BP}$ were non-BP cases. All of these subjects were disabled inpatients because of cerebral stroke, etc. All BP cases were diagnosed by a dermatologist and had anti-BP 180 antibody. We assessed the clinical variables as follows: age, sex, underling disease of hospitalization, length of hospital stay, feeding method, medication, tracheal tube indwelling and frequency of death and compared the 2 groups with and without BP.

In the results, "age" indicated the age as of April 30, 2009 or death age. Main feeding method was defined as the method of nutrient supply with the longest duration during the hospital stay. Percent length of feeding method was the proportion of length of each feeding method to the whole hospital stay (\%). Because some cases underwent 2 or more feeding methods at the same time, the sum of the length or percentage of each method exceeded whole hospital stay or $100 \%$. Since some drugs such as aldosterone antagonist and neuroleptics have been reported to have a possible relation to BP (5), administration of these drugs was also checked.

\section{Statistical Analysis}

The Pearson chi square test or Student's t-test was used for the statistical analysis of comparisons of two groups. Univariate analysis (Fischer's exact test) was performed with variables such as age, gender, length of hospital stay, feeding method and its duration (including percent length), drug, indwelling tracheal tube and death in order to determine the factors associated with BP. Multivariate analysis by logistic regression was done to determine which variables were independently associated with BP. Factors that were at least marginally associated with $\mathrm{BP}$ in univariate analysis $(\mathrm{p}<$ $0.05)$ together with age and sex which were reported to be common feature of BP (3), were tested. For this analysis, we reported the Nagelkerke $\mathrm{R}^{2}$ as a measure of the predictive relationship among variables. Multivariate analysis by Cox proportional hazard regression was also performed to quantify risk for BP. The cumulative incidence of BP over time was estimated by Kaplan-Meier method and was compared with use of the log-rank tests.

A $p$ value of less than 0.05 was considered to be significant. The SPSS (SPSS Inc., Chicago, IL) and Statistica (StatSoft Inc., Tulsa, OK) were used for all statistical computations.

\section{Results}

Clinical characteristics are summarized in Table 1. All of the subjects were elderly persons, and the main reason for hospitalization was disability due to cerebral damage resulting from stroke or other neural diseases. Age and length of hospital stay did not differ between the groups. Most BP patients were females in contrast to non-BP cases but the ratio of males to females was not significantly different. Main feeding method of BP patients was enteral feeding by percu- taneous endoscopic gastrostomy (PEG, 60\%) and nasogastric (NG) tube (40\%), and none of the patients underwent other methods. On the other hand, in the non-BP group, $42 \%$ of subjects were fed by tube feeding and $39 \%$ could do peroral feeding. The frequency of tube (PEG and NG) and PEG feeding were significantly greater in the BP group as compared to the non-BP group. The length of each feeding method was not different between the two groups, but percent length of tube feeding to whole hospital stay was significantly greater in the BP group. The frequencies of taking aldosterone antagonist and neuroleptics were not different between the groups. Frequencies of indwelling tracheal tube and death were not different. The main cause of death was infection, which had the same tendency in both groups.

Skin lesions such as serous blisters were initially observed on the trunk, legs or arms and then spread over the whole body (Fig. 1). Eosinophilia was also observed in all cases. Mucous membranes were affected in 2 out of 5 cases and one case had esophageal mucosal desquamation which induced hematemesis. All of the patients were treated by systemic administration of prednisolone at an initial dose of $0.6-0.8 \mathrm{mg} / \mathrm{kg} / \mathrm{day}$, which was reduced gradually, but none of the cases could stop it because of recurrence of blisters associated with reducing the dose in their lifetime during the survey period. These clinical features were not different from those of common BP cases.

In the univariate analysis, among the following variables such as age, sex, length of hospitalization, main feeding method, length and percent length of each feeding method, drug, indwelling tracheal tube and death, PEG $(\mathrm{p}=0.04)$ and tube (PEG and NG) feeding $(\mathrm{p}=0.045)$ as main feeding method were associated with BP.

The multivariate logistic analysis was then performed with age, sex and PEG feeding. Among these factors, PEG was significantly and independently associated with $\mathrm{BP}\left(\mathrm{R}^{2}=\right.$ 0.403, Table 2). However, when adding NG tube feeding to PEG one, i.e. variable enteral tube feeding (PEG and NG) was not associated.

The mean duration of tube feeding until the onset of BP

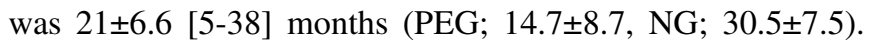
All the onset of BP followed the initiation of enteral feeding. Then multivariate analysis by Cox proportional hazard regression was also performed with age, sex and PEG. In this analysis, Day 0 was defined as the beginning day of the main nutrition method in each subject. In PEG, NG tube feeding or total parenteral nutrition, Day 0 was nearly equal to the date of PEG operation, tube placement or central venous catheterization, respectively. Study end date for BP patients was defined as date of the onset of BP. Study end date for non-BP patients was defined as date for death, date for changing main nutrition method to others, or as of April 30, 2009. It demonstrated PEG was significantly associated with increased risk for $\mathrm{BP}(\mathrm{p}=0.014$, hazard ratio $=11.7 ; 95 \%$ confidence interval=1.64-83.3).

The cumulative incidence of BP over time in the patients with PEG or with other nutrition methods is shown in 
Table 1. Characteristics of the Subjects

\begin{tabular}{|c|c|c|c|}
\hline & $\mathrm{BP}(\mathrm{N}=5)$ & $\begin{array}{l}\text { Non-BP } \\
(\mathrm{N}=31)\end{array}$ & $\mathrm{p}$ value \\
\hline Age & $87.8 \pm 3.2$ & $83.3 \pm 1.7$ & 0.31 \\
\hline Sex M/F & $1 / 4$ & $19 / 12$ & 0.094 \\
\hline \multicolumn{4}{|l|}{ Causative disease of hospitalization N (\%) } \\
\hline Cerebral infarction & $4(80)$ & $17(54.8)$ & 0.30 \\
\hline Congestive heart failure & & $4(12.9)$ & 0.40 \\
\hline Cerebral hemorrhage & $1(20)$ & $3(9.7)$ & 0.50 \\
\hline Chronic respiratory failure & & $3(9.7)$ & 0.46 \\
\hline Encephalitis & & $2(6.5)$ & 0.56 \\
\hline Spinocerebellar degeneration & & $1(3.2)$ & 0.69 \\
\hline Parkinson disease & & $1(3.2)$ & 0.69 \\
\hline Length of hospital stay (months) & $39.2 \pm 6.2$ & $42.7 \pm 3.2$ & 0.68 \\
\hline \multicolumn{4}{|l|}{ Main feeding method N (\%) } \\
\hline Tube feeding (PEG + NG tube) & $5(100)$ & $13(42)$ & 0.022 \\
\hline PEG & $3(60)$ & $4(13)$ & 0.019 \\
\hline NG tube & $2(40)$ & $9(29)$ & 0.62 \\
\hline TPN & $0(0)$ & $6(19)$ & 0.29 \\
\hline Peroral feeding & $0(0)$ & $12(39)$ & 0.096 \\
\hline Length of tube feeding (months) & $34.4 \pm 6.7$ & $19.9 \pm 4.7$ & 0.24 \\
\hline PEG & $18.2 \pm 8.5$ & $7.6 \pm 3.4$ & 0.26 \\
\hline NG tube & $16.2 \pm 10.9$ & $12.2 \pm 4.1$ & 0.72 \\
\hline Length of TPN (months) & $4.8 \pm 2.0$ & $7.0 \pm 2.1$ & 0.69 \\
\hline Length of peroral feeding (months) & $0.2 \pm 0.2$ & $15.8 \pm 3.8$ & 0.11 \\
\hline $\begin{array}{l}\text { Percent length of tube feeding to whole } \\
\text { hospital stay (\%) }\end{array}$ & $86.2 \pm 6.7$ & $42.4 \pm 8.0$ & 0.038 \\
\hline PEG & $46.2 \pm 19.2$ & $17.2 \pm 6.6$ & 0.11 \\
\hline NG tube & $40.0 \pm 24.5$ & $25.2 \pm 7.0$ & 0.46 \\
\hline $\begin{array}{l}\text { Percent length of TPN to whole hospital } \\
\text { stay }(\%)\end{array}$ & $13.8 \pm 6.7$ & $19.2 \pm 6.0$ & 0.73 \\
\hline $\begin{array}{l}\text { Percent length of peroral feeding to whole } \\
\text { hospital stay }(\%)\end{array}$ & $0.4 \pm 0.4$ & $38.5 \pm 8.5$ & 0.079 \\
\hline \multicolumn{4}{|l|}{ Drug N (\%) } \\
\hline Aldosterone antagonist & $0(0)$ & $6(19)$ & 0.29 \\
\hline Neuroleptics & $1(20)$ & $1(3.2)$ & 0.14 \\
\hline Tracheal tube N (\%) & $2(40)$ & $11(35)$ & 0.83 \\
\hline Died N (\%) & $4(80)$ & $15(48)$ & 0.19 \\
\hline \multicolumn{4}{|l|}{ Cause of death N (\%) } \\
\hline Sepsis & $2(50)$ & $6(40)$ & 0.68 \\
\hline Pneumonia & $2(50)$ & $6(40)$ & 0.68 \\
\hline Congestive heart failure & & $2(13.3)$ & 0.39 \\
\hline Common bile duct cancer & & $1(6.7)$ & 0.56 \\
\hline
\end{tabular}

N, number; BP, bullous pemphigoid; M, male; F, female; PEG, percutaneous endoscopic gastrostomy; NG, nasogastric; TPN, total parenteral nutrition

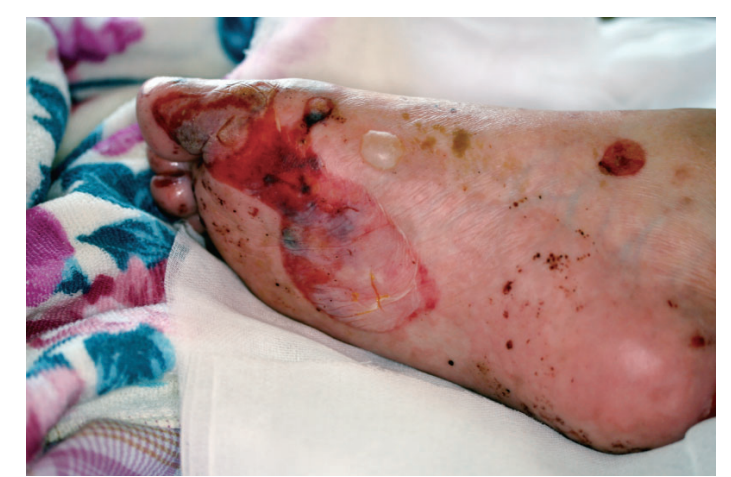

Figure 1. Clinical presentation of bullous pemphigoid. Tense, partly hemorrhagic serous blisters and erosions were observed.

Fig. 2. It was significantly higher in the patients with PEG than ones without PEG ( $\mathrm{p}=0.010$, log-rank test).

\section{Discussion}

$\mathrm{BP}$ is a rare disease in the general population. Previous 
Table 2. Factors Associated with BP: Results of a Multivariate Logistic Analysis

\begin{tabular}{|c|c|c|c|c|c|}
\hline Variable & \multicolumn{5}{|c|}{ Regression coefficient } \\
\hline & $\begin{array}{c}\text { Estimated } \\
\text { value }\end{array}$ & $\begin{array}{c}\text { Standard } \\
\text { error }\end{array}$ & p value & Odds ratio & $95 \%$ CI \\
\hline Age & 0.0282 & 0.0733 & 0.700 & 1.03 & $0.891-1.19$ \\
\hline Sex & -2.34 & 1.45 & 0.107 & 0.0968 & $0.0057-1.65$ \\
\hline $\begin{array}{c}\text { PEG } \\
\text { feeding }\end{array}$ & 2.80 & 1.32 & 0.0345 & 16.4 & $1.23-220$ \\
\hline
\end{tabular}

CI denotes confident interval. Sex; male $=1$, Female $=0$. PEG feeding; PEG $=1$, other feeding method $=0$. Nagelkerke $\mathrm{R}^{2}=0.403$.

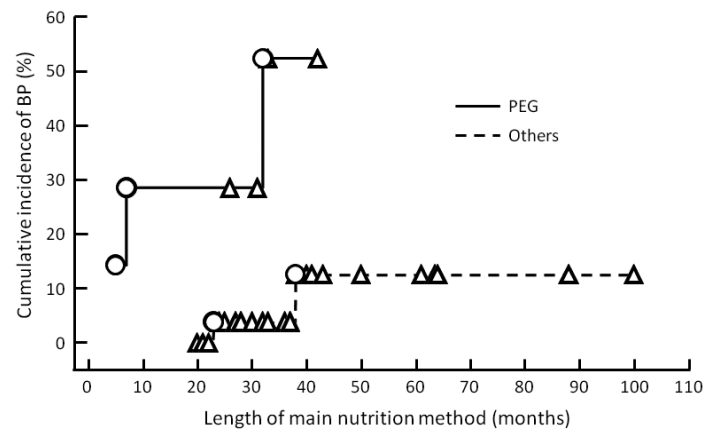

Figure 2. Kaplan-Meier survival curves. These curves showed that the cumulative incidence of BP was significantly higher in the patients with PEG than in those without PEG (p=0.010, log-rank test). $\bigcirc$; BP case. $\triangle$; Patient who did not experience BP.

studies of BP have reported incidences between 0.2 and 3 per 100,000 person years (6-8). A recent large cohort survey in United Kingdom demonstrated the highest incidence, 4.3 per 100,000 person years. Moreover, this survey also reported that the frequency was increasing lately, i.e. 4.8-fold over the 11 years from 1996 to 2006 (3). Although the reason for this increase is not known, this disease may become not so rare in the near future. On the other hand, the incidence was reported to be relatively high among elderly in nursing homes, i.e. 4.8 per 100 person years (4). We detected 5 BP cases among 36 hospitalized patients with a long stay for 46 months. The crude incidence was calculated to be 3.6 per 100 inpatient years, which is comparable to that mentioned above. These lines of evidence suggest that BP is pretty common in institutionalized elders as compared to the general population.

Skin blisters, particularly localized blisters, are frequently observed among disabled elders in the genitoinguinal area or skin near catheter, which are commonly thought to result from diaper and catheter contact or friction. However, localized BP is not rare and the cases with BP the lesions of which began in the skin near catheter or the ones which were initially diagnosed as contact dermatitis have also been reported $(9,10)$. The diagnosis of localized BP is commonly delayed (11). Moreover, Fernandez-Viadero et al demonstrated that 17 (59\%) of 29 elders in a nursing home having blisters were pathologically diagnosed as BP (4). In this context, BP may be often misdiagnosed as other blistering diseases. The institutionalized elders are frequently disabled, so that they cannot easily visit a dermatologist, which may be also one of the reasons for the misdiagnosis. Since the management of these persons is principally performed by physicians, greater awareness of this disease is needed for physicians.

The most important point of our study is that PEG was found to be an independent risk factor for BP. The meaning of this phenomenon is not known. BP is an autoimmune disease but it was reported to be induced by variety of trauma events to the skin $(10,12)$. Cases with BP localized to the skin around a urostomy or colostomy site were also reported $(10,13)$. These cases are thought to be directly related to the skin damage at ostomy surgery. The damage to dermo-epidermal junction with subsequent antigen exposure and activation of the immune system leading to the production of autoantibody might be involved with this association (14). Skin damage also occurred at PEG, but all of our BP cases had widespread lesions, moreover, blisters did not develop initially in the skin around PEG. However, it does not deny this hypothesis, because if the autoantibody systemically circulated and acted, the lesions would develop in any area of skin. On the other hand, indwelling tracheal tube was not associated with BP even though skin damage occurred at tracheotomy, suggesting that PEG may have some special meaning on the pathogenesis. The skin around the PEG tube is frequently inflamed by gastric juice and chronically irritating skin may induce activation of the immune system, which is the possible difference between PEG and tracheal tube.

Although NG tube feeding was not associated statistically, all of the patients underwent feeding with enteral formula. Many autoimmune disorders including BP are occasionally induced by drugs (5). In this context, we cannot deny the possibility that some substances contained in enteral formula may induce dysregulation of the immunological control system observed in BP. The total amount of calories by enteral tube feeding was 1,200-1,400 Kcal with $60 \mathrm{~g}$ protein and 25 g lipids, which was thought to be sufficient for bedridden old persons, because under this nutrition, significant body weight decrease was not observed. The body mass index at the onset of BP was $21.9 \pm 1.5$ and it was $19.8 \pm 0.46$ in non$\mathrm{BP}$ cases, which were not different between groups, suggesting malnutrition may not be associated with BP.

The present study has several limitations. Sample size is 
small and the retrospective survey conducted at the single institution does not have strong power. It should be still premature to generalize this finding to other populations. However, even though the number of patients analyzed was small, the present study demonstrated PEG was a significant risk factor for BP, suggesting this result should not be ignored. Since several commercially supplied enteral formula and feeding tubes were used in this study, we should notice these variations to understand the mechanism of this association and further investigation is needed.

In summary, we have shown that PEG was a significant risk factor for BP. Since only a small number of patients was retrospectively surveyed in the present study, large-scale clinical prospective studies in multiple medical centers would be warranted to confirm this finding in the near future. Because PEG has become the primary choice to establish enteral access for disabled elders unable to take oral feedings lately, the frequency of BP may increase more in the near future. BP is not such a rare disease among the long stay hospitalized elders and it may be frequently misdiagnosed. Since physicians manage these patients with PEG, a greater awareness of this disease is needed.

\section{References}

1. Liu Z, Diaz LA, Troy JL, et al. A passive transfer model of the organ-specific autoimmune disease, bullous pemphigoid, using antibodies generated against the hemidesmosomal antigen, BP180. J Clin Invest 92: 2480-2488, 1993.

2. Stanley JR, Hawley-Nelson P, Yuspa SH, Shevach EM, Katz SI. Characterization of bullous pemphigoid antigen: a unique basement membrane protein of stratified squamous epithelia. Cell $\mathbf{2 4}$ 897-903, 1981.

3. Langan SM, Smeeth L, Hubbard R, Fleming KM, Smith CJ, West J. Bullous pemphigoid and pemphigus vulgaris--incidence and mortality in the UK: population based cohort study. BMJ 337: a180, 2008.

4. Fernandez-Viadero C, Arce Mateos F, Verduga Velez R, Crespo Santiago D. Blisters in a nursing home: bullous pemphigoid more often than we think? J Am Geriatr Soc 52: 1405-1406, 2004.

5. Bastuji-Garin S, Joly P, Picard-Dahan C, et al. Drugs associated with bullous pemphigoid. A case-control study. Arch Dermatol 132: 272-276, 1996.

6. Adam BA. Bullous diseases in Malaysia: epidemiology and natural history. Int J Dermatol 31: 42-45, 1992.

7. Garcia-Doval I, Mayo E, Nogueira Farina J, Cruces MJ. Bullous pemphigoid triggered by influenza vaccination? Ecological study in Galicia, Spain. Br J Dermatol 155: 820-823, 2006.

8. Jung M, Kippes W, Messer G, Zillikens D, Rzany B. Increased risk of bullous pemphigoid in male and very old patients: A population-based study on incidence. J Am Acad Dermatol 41: 266-268, 1999.

9. Kirtschig G, Walkden VM, Venning VA, Wojnarowska F. Bullous pemphigoid and multiple sclerosis: a report of three cases and review of the literature. Clin Exp Dermatol 20: 449-453, 1995.

10. Salomon RJ, Briggaman RA, Wernikoff SY, Kayne AL. Localized bullous pemphigoid. A mimic of acute contact dermatitis. Arch Dermatol 123: 389-392, 1987.

11. Tran JT, Mutasim DF. Localized bullous pemphigoid: a commonly delayed diagnosis. Int J Dermatol 44: 942-945, 2005.

12. Dahl MG, Cook LJ. Lesions induced by trauma in pemphigoid. $\mathrm{Br}$ J Dermatol 101: 469-473, 1979.

13. Torchia D, Caproni M, Ketabchi S, Antiga E, Fabbri P. Bullous pemphigoid initially localized around a urostomy. Int J Dermatol 45: 1387-1389, 2006.

14. Downs AM, Lear JT, Bower CP, Kennedy CT. Does influenza vaccination induce bullous pemphigoid? A report of four cases. $\mathrm{Br}$ J Dermatol 138: 363, 1998.

(C) 2010 The Japanese Society of Internal Medicine http://www.naika.or.jp/imindex.html 